\title{
Late-Onset Glaucoma-Filtrating Bleb Leak in a Penetrating Keratoplasty Patient: A Case Report
}

\author{
Zuleyha Yalniz-Akkaya, Ayse Burcu, and Firdevs Ornek \\ Ophthalmolgy Clinic, Ministry of Health Ankara Training and Research Hospital, TR06340 Ankara, Turkey \\ Correspondence should be addressed to Zuleyha Yalniz-Akkaya, zyalniz@yahoo.com
}

Received 27 November 2011; Accepted 2 February 2012

Academic Editor: C.-Y. Cheng

Copyright (๑) 2012 Zuleyha Yalniz-Akkaya et al. This is an open access article distributed under the Creative Commons Attribution License, which permits unrestricted use, distribution, and reproduction in any medium, provided the original work is properly cited.

\begin{abstract}
Introduction. Late-onset bleb leaks occur more frequently after the use of adjunctive antimetabolites and require surgical management to seal and preserve filtrating bleb. Case Presentation. A 48-year-old female presented with decreased visual acuity for five days in her left eye. She had a left penetrating keratoplasty one year earlier and two trabeculectomies 7 years earlier. Visual acuity was hand motions, intraocular pressure was $3 \mathrm{mmHg}$, corneal graft was clear, mature cataract was present, and axial length was $30.48 \mathrm{~mm}$. The conjunctiva covering the superotemporal sclerotomy was avascular, flat, and partially lost. After heavily painting the bleb with a fluorescein, late-onset point leak was revealed. Overlying conjunctiva was excised. The atrophic, irregular, and partially absent scleral flap was covered by a processed human pericardium graft and conjunctival advancement. Postoperatively, intraocular pressure stabilized around $16 \mathrm{mmHg}$. After four months, phacoemulsification and intraocular lens implantation were performed. Visual acuity did not exceed 0.1 (in decimal notation) due to degenerative myopia-related macular atrophy. Corneal graft remained clear at her 6-month followup period. Conclusion. Surgical bleb revision using a pericardium graft and conjunctival advancement seems to be an effective method for treating late bleb leaks. However, careful follow-up is required for detecting recurrent leaks and elevated intraocular pressure.
\end{abstract}

\section{Introduction}

Late-onset bleb leaks are defined as leaks occurring more than three months after trabeculectomy. Although the use of antimetabolites (mitomycin C and 5-fluorouracil) has increased the success rate of trabeculectomy, the incidence of late bleb leaks also increased along with other complications [1]. For the treatment of late bleb leaks, surgical intervention is necessary and various techniques have been described in previous reports.

\section{Case Report}

A 48-year-old female patient called to the Cornea Department prior to her appointment complaining of decreased visual acuity (VA) in her left eye for five days. She underwent left penetrating keratoplasty for nonspecific corneal leucoma one year earlier. Approximately 7 years earlier, she underwent left trabeculectomy twice, at a different institution. Data on the use of adjunctive antimetabolites was not available.

Visual acuity was measured using a Snellen chart. Intraocular pressure (IOP) was measured by a Goldmann applanation tonometer. Ocular examination was performed using a slit lamp biomicroscopy. Bleb leak was checked under cobalt blue slit-lamp illumination after staining with flourescein.

Over the last three months, VA decreased in her left eye from "counting fingers at one meter" to "hand motions," the IOP decreased from $17 \mathrm{mmHg}$ to $3 \mathrm{mmHg}$. The nuclear sclerosis (grade III) and posterior subcapsular cataract progressed to a mature cataract (Figure 1). Additionally, three sclerotomies (superonasally, superiorly, and superotemporally) and two iridectomies (superonasally and superotemporally) were noted. The conjunctiva covering the superotemporal sclerotomy was avascular, flat, and partially lost (Figure 1). The corneal graft was clear and secured with 


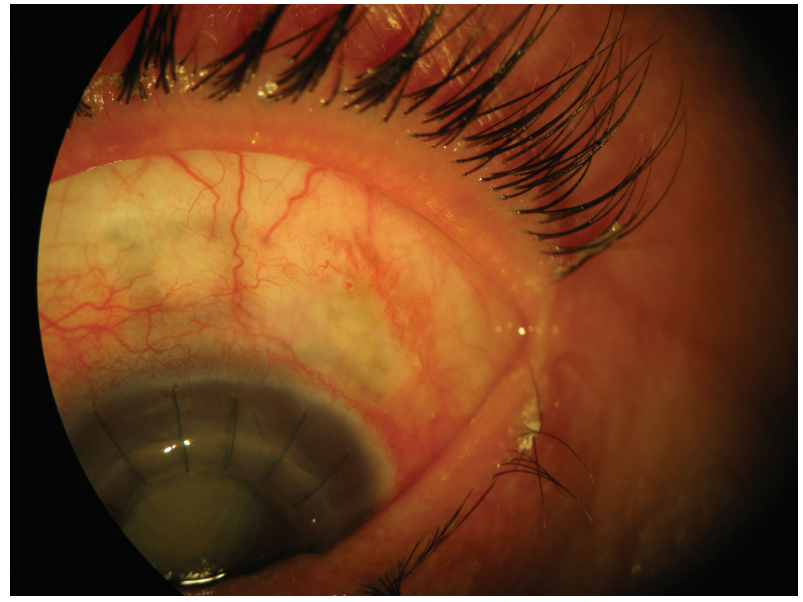

FIGURE 1: The avascular conjunctiva covering the superotemporal sclerotomy.

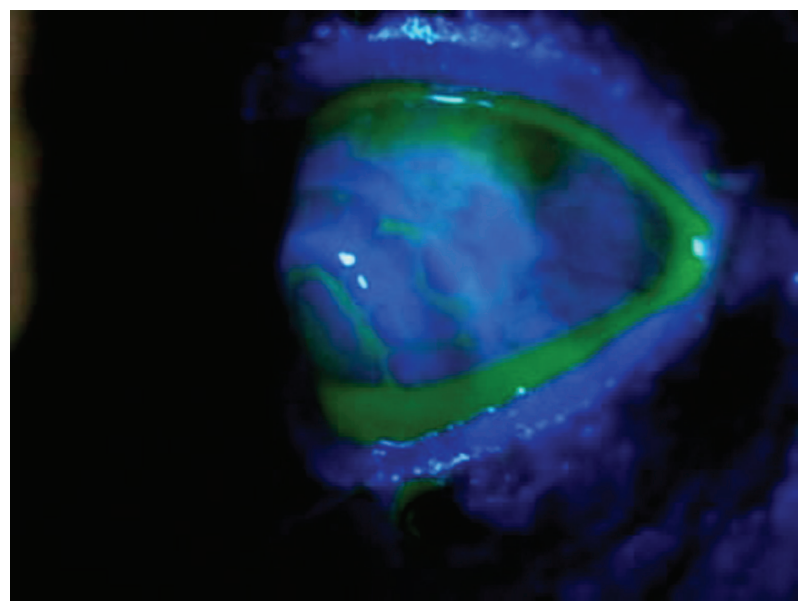

Figure 2: The leakage streaming down.

16 interrupted 10-0 nylon sutures. B-scan ultrasonographic evaluation of the posterior segment was normal. Axial length was $30.48 \mathrm{~mm}$. After an unsuccessful first attempt to identify a bleb leak with $2 \%$ fluorescein, we were able to observe the Siedel positivity with a heavily painted bleb and we made the diagnosis of late-onset filtrating bleb leak (Figure 2) which was a point leak but not an oozing.

In her right eye, the penetrating corneal graft was clear (for 27 years), distant corrected visual acuity was 0.5 (in decimal notation), IOP was $12 \mathrm{mmHg}$ (with dorzolamide and timolol maleate combination, (Cosopt, Merck \& Co. Inc., NJ, USA)). Axial length was $30.20 \mathrm{~mm}$. Posterior segment was consistent with degenerative myopia.

In order to eliminate the risk of infection and obtain adequate IOP, surgical intervention was scheduled to fix the leak. The surgical procedure was performed under topical and subconjunctival anesthesia. The avascular conjunctiva was excised together with $1 \mathrm{~mm}$ of healthy conjunctiva. An atrophic and irregular scleral flap was exposed. The surrounding relatively healthy conjunctiva was firmly attached

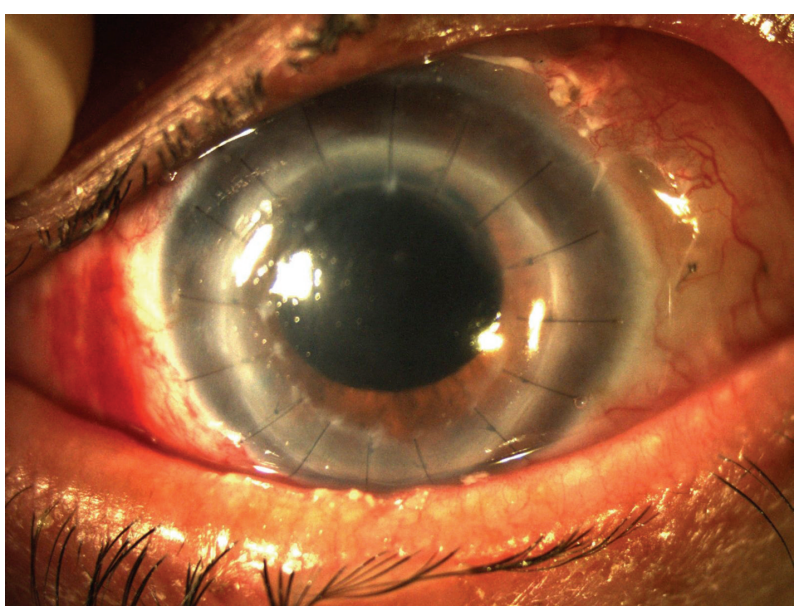

FIgure 3: Postoperative picture: elevated filtrating bleb and cataract removed.

to the underlying sclera, causing difficult subconjunctival dissection. As the scleral flap could not be fixed properly and adequate sclera for flap creation was not available due to the firm subconjunctival scarring and degenerative myopia-associated thin sclera, the decision was made to overlay the atrophic and partially absent scleral flap with a processed human pericardium patch graft (Tutopatch, Tutogen Medical GmbH, Neunkirchen am Brand, Germany) followed by conjunctival advancement. Four interrupted 100 nylon (Daclon, Smi, St. Vith, Belgium), sutures were used to secure the graft. The tightness was adjusted so as to allow aqueous drainage underneath the graft and not to seal it completely. After a large subconjunctival dissection, the conjunctiva was advanced and closed with multiple 100 nylon sutures. The limbal side was closed with running mattress suture, and watertight wound closure was obtained with minimal tension. Ofloxacin (Exocin, Allergan Pharmaceuticals Inc., County Mayo, Ireland) and dexamethasone sodium phosphate (Maxidex, Alcon Laboratories Inc., TX, USA) were used eight times per day postoperatively for one week and one month, respectively.

The IOP stabilized at $16 \mathrm{mmHg}$ without medication, and a functioning filtrating bleb was formed (Figure 3). After four months, phacoemulsification and a +7.00 D intraocular lens (Acrysof MA60AC, Alcon Laboratories Inc, TX, USA) implantation were performed. Visual acuity did not exceed 0.1 (in decimal notation) due to degenerative myopia. Although macular edema was not detected after the cataract surgery, hypotony-associated macular edema sequela could have been another cause for low final visual acuity. The corneal graft remained clear at her 6-month followup, after the phacoemulsification (Figure 3 ).

\section{Discussion}

This is the second reported case of late bleb leak in a penetrating keratoplasty patient. The first one was a patient that developed bleb leak after combined penetrating keratoplasty and trabeculectomy with mitomycin C [2]. According to 
reports published to date, the prevalence of late-onset bleb leaks was reported to range between $1.4 \%$ and $14.6 \%$ with adjunctive antimetabolite application $[1,3]$. Late bleb leaks can occur from three months to years after surgery; the reported mean appearance time was 27.9 months [3].

The goal of treatment is to seal the leak, eliminate the hypotony, and maintain the target IOP by preserving a functional filtering bleb. As an established treatment method is not present, several treatments have been described for the management of late bleb leaks with various success rates. Nonincisional treatments consist of observation with or without aqueous suppression, pressure patches, collagen shields, bandage contact lenses, autologous serum drops, intrableb autologous blood injections, Holmium laser bleb revision, Neodymium: Yttrium Aluminium Garnet laser bleb remodeling, Argon laser "spot-welding," cyanoacrylate, and fibrin tissue adhesives [4]. Incisional treatments consist of conjunctival advancement or rotational flaps, free conjunctival flaps and bleb excision with amniotic membrane transplantation, patching with processed donor collagenous tissue (e.g., sclera, pericardium, or dura mater), and corneal stromal patch graft. These incisional procedures can be associated with or without bleb excision [4-7]. Incisional treatments were reported to be more effective than nonincisional treatments with respect to leak resolution [8]. The reported success rates are up to $93 \%[9,10]$. However, additional antiglaucomatous treatment can be required in up to $60 \%$ of patients [8-10]. After closing the trabeculectomy site with a scleral graft, Miller et al. implanted a Baerveldt Glaucoma Device to maintain drainage and eliminate the possibility of postoperative IOP rise [5].

Our patient had a leaking bleb, hypotony, hypotonyassociated cataract, previous penetrating keratoplasty, and degenerative myopia. She was treated successfully with donor pericardium graft, conjunctival advancement, and cataract removal. In spite of the short-term successful treatment, corneal graft decompensation, recurrence of leakage, and elevation of IOP are potential long-term risks requiring further medical or surgical interventions.

The bleb should be routinely checked for leaks, as lateonset bleb leaks can lead to serious complications, and early diagnosis is critical for successful treatment. Fluorescein strips should be the preferred method in order to paint the bleb and reveal occult leaks.

\section{Conclusion}

Surgical bleb revision with pericardium patch graft and conjunctival advancement seems to be an effective method for treating late-onset bleb leaks. However, careful followup is required for detecting and intervening on recurrent leaks and elevated IOP.

\section{Disclosure}

This study has not been published, accepted for publication, or is not currently under consideration, in whole or in part, in English or in another language, elsewhere. The authors have not used any sources from public or private financial support. The authors have no proprietary or commercial interest in any materials discussed in this paper. All the authors read the paper and agreed on its publication in this form. The paper was edited for language correction (http:// www.editavenue.com/, file number: EA305205923).

\section{References}

[1] D. S. Greenfidd, J. M. Liebmann, J. Jee, and R. Ritch, "Lateonset bleb leaks after glaucoma filtering surgery," Archives of Ophthalmology, vol. 116, no. 4, pp. 443-447, 1998.

[2] D. WuDunn, E. Alfonso, and P. F. Palmberg, "Combined penetrating keratoplasty and trabeculectomy with mitomycin C," Ophthalmology, vol. 106, no. 2, pp. 396-400, 1999.

[3] R. Bindlish, G. P. Condon, J. D. Schlosser, J. D’Antonio, K. B. Lauer, and R. Lehrer, "Efficacy and safety of mitomycin-C in primary trabeculectomy: five-year follow-up," Ophthalmology, vol. 109, no. 7, pp. 1336-1341, 2002.

[4] R. M. Feldman and G. Altaher, "Management of late-onset bleb leaks," Current Opinion in Ophthalmology, vol. 15, no. 2, pp. 151-154, 2004.

[5] G. Miller, D. Y. Tam, and I. I. K. Ahmed II, "Late bleb leak management with trabeculectomy shutdown, baerveldt glaucoma device implantation, and titratable tube lumen size," Journal of Glaucoma, vol. 20, no. 1, pp. 51-56, 2011.

[6] N. Harizman, R. Ben-Cnaan, M. Goldenfeld, H. LevkovitchVerbin, and S. Melamed, "Donor scleral patch for treating hypotony due to leaking and/or overfiltering blebs," Journal of Glaucoma, vol. 14, no. 6, pp. 492-496, 2005.

[7] A. Mistlberger, R. Biowski, and G. Grabner, "Repair of a lateonset filtering bleb leak using a corneal graft shaped with an excimer laser," Ophthalmic Surgery and Lasers, vol. 32, no. 5, pp. 428-431, 2001.

[8] A. L. Burnstein, D. WuDunn, S. L. Knotts, Y. Catoira, and L. B. Cantor, "Conjunctival advancement versus nonincisional treatment for late-onset glaucoma filtering bleb leaks," Ophthalmology, vol. 109, no. 1, pp. 71-75, 2002.

[9] D. P. Tannenbaum, D. Hoffman, M. J. Greaney, and J. Caprioli, "Outcomes of bleb excision and conjunctival advancement for leaking or hypotonous eyes after glaucoma filtering surgery," British Journal of Ophthalmology, vol. 88, no. 1, pp. 99-103, 2004.

[10] D. L. Budenz, P. P. Chen, and Y. K. Weaver, "Conjunctival advancement for late-onset filtering bleb leaks: indications and outcomes," Archives of Ophthalmology, vol. 117, no. 8, pp. 1014-1019, 1999. 


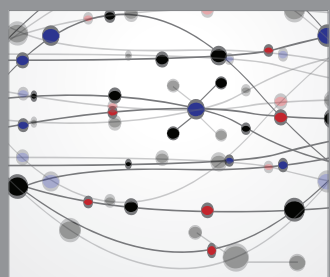

The Scientific World Journal
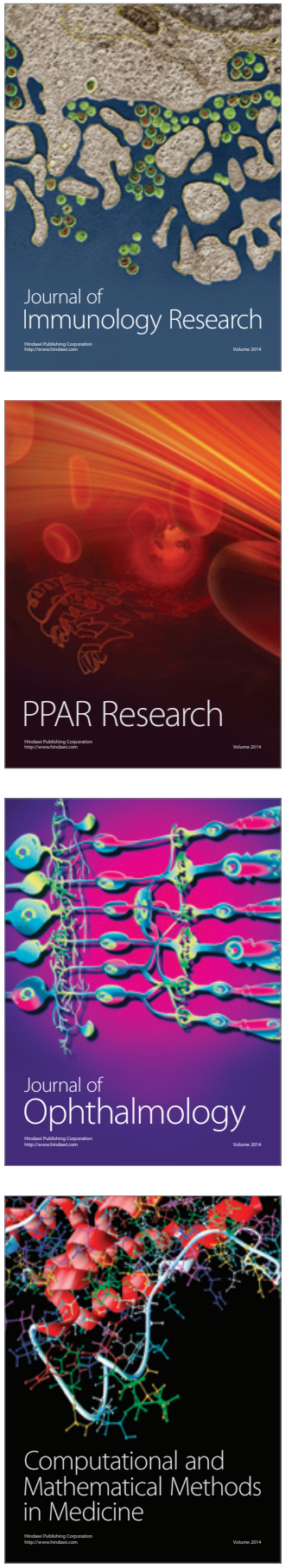

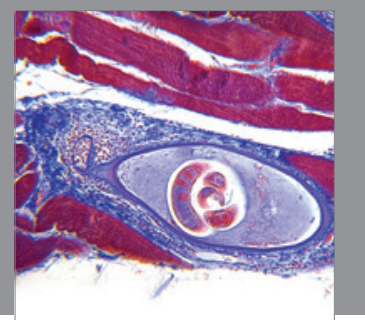

Gastroenterology

Research and Practice
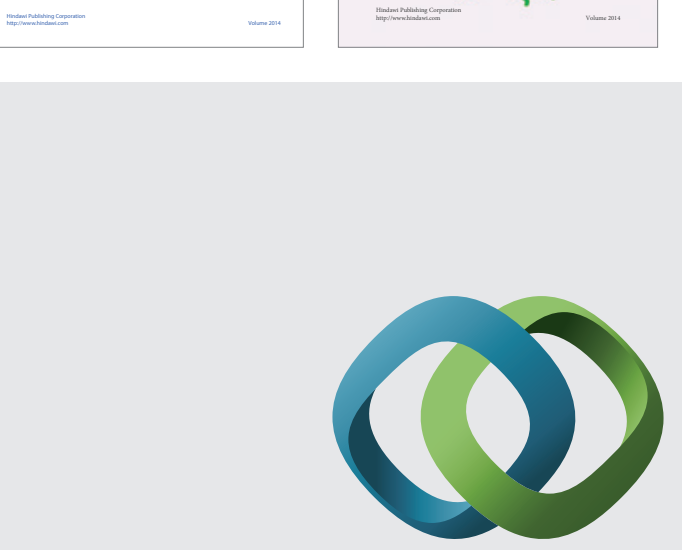

\section{Hindawi}

Submit your manuscripts at

http://www.hindawi.com
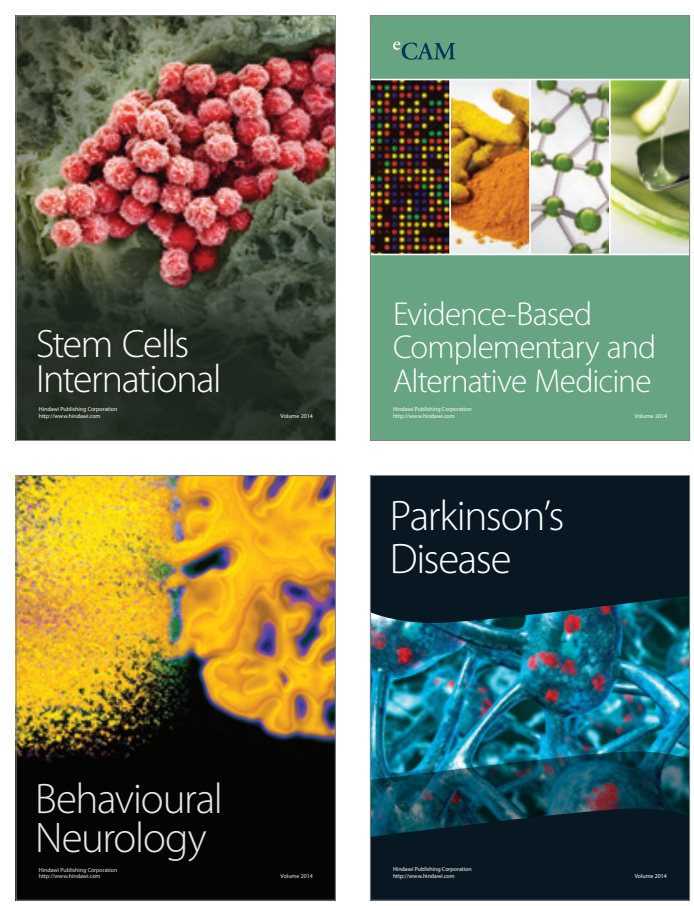

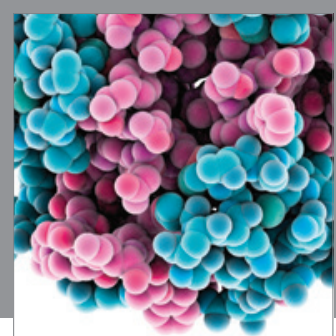

Journal of
Diabetes Research

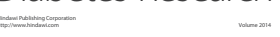

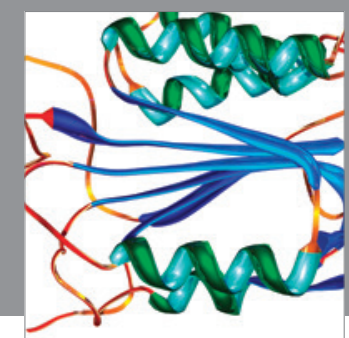

Disease Markers
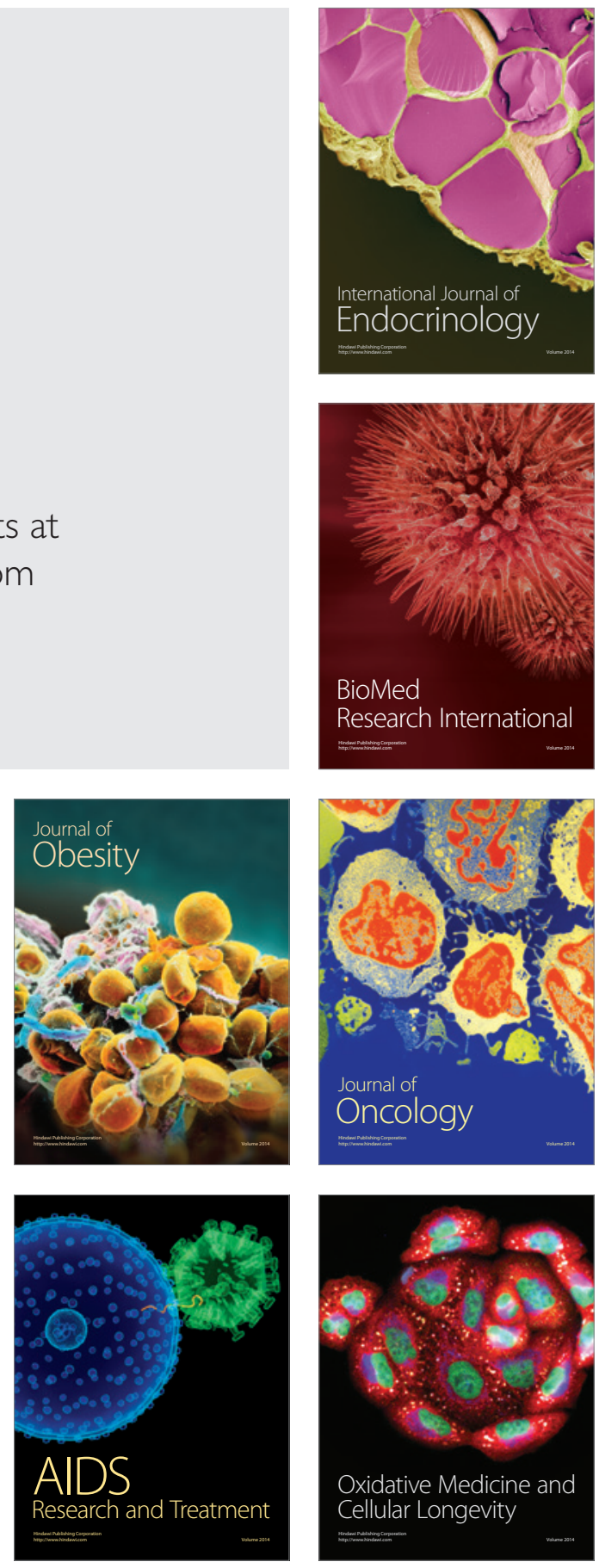